Anomalous transport effects on switching currents of graphene-based Josephson junctions

This content has been downloaded from IOPscience. Please scroll down to see the full text.

2017 Nanotechnology 28134001

(http://iopscience.iop.org/0957-4484/28/13/134001)

View the table of contents for this issue, or go to the journal homepage for more

Download details:

IP Address: 193.205.162.75

This content was downloaded on 27/02/2017 at 14:28

Please note that terms and conditions apply. 


\title{
Anomalous transport effects on switching currents of graphene-based Josephson junctions
}

\author{
Claudio Guarcello ${ }^{1,2,3}$, Davide Valenti ${ }^{4}$, Bernardo Spagnolo ${ }^{3,4,5}$, \\ Vincenzo Pierro ${ }^{6}$ and Giovanni Filatrella ${ }^{7}$ \\ ${ }^{1}$ SPIN-CNR, Via Dodecaneso 33, I-16146 Genova, Italy \\ ${ }^{2}$ NEST, Istituto Nanoscienze-CNR and Scuola Normale Superiore, Piazza S. Silvestro 12, I-56127 Pisa, \\ Italy \\ ${ }^{3}$ Radiophysics Department, Lobachevsky State University, Nizhniy Novgorod, Russia \\ ${ }^{4}$ Dipartimento di Fisica e Chimica, Group of Interdisciplinary Theoretical Physics, Università di Palermo \\ and CNISM, Unità di Palermo, Palermo, Italy \\ ${ }^{5}$ Istituto Nazionale di Fisica Nucleare, Sezione di Catania, Catania, Italy \\ ${ }^{6}$ Dipartimento di Ingegneria, Università del Sannio, Benevento, Italy \\ ${ }^{7}$ Dipartimento di Scienze e Tecnologie and CNISM, Unità di Salerno, Università del Sannio, Benevento, \\ Italy
}

E-mail: claudio.guarcello@unipa.it

Received 3 January 2017, revised 2 February 2017

Accepted for publication 6 February 2017

Published 27 February 2017

\begin{abstract}
We explore the effect of noise on the ballistic graphene-based small Josephson junctions in the framework of the resistively and capacitively shunted model. We use the non-sinusoidal currentphase relation specific for graphene layers partially covered by superconducting electrodes. The noise induced escapes from the metastable states, when the external bias current is ramped, given the switching current distribution, i.e. the probability distribution of the passages to finite voltage from the superconducting state as a function of the bias current, that is the information more promptly available in the experiments. We consider a noise source that is a mixture of two different types of processes: a Gaussian contribution to simulate an uncorrelated ordinary thermal bath, and non-Gaussian, $\alpha$-stable (or Lévy) term, generally associated to nonequilibrium transport phenomena. We find that the analysis of the switching current distribution makes it possible to efficiently detect a non-Gaussian noise component in a Gaussian background.
\end{abstract}

Keywords: graphene, Josephson junctions, Lévy processes, non-thermal noise

(Some figures may appear in colour only in the online journal)

\section{Introduction}

Josephson junction ( $\mathrm{JJ}$ ) electrical behavior is governed by a quantum variable, the gauge invariant phase difference between the macroscopic phases of the two superconductors forming the junction. Its dynamics, as described by the celebrated Josephson equations [1, 2], is not directly accessible, and only indirect electrical measurements (essentially, the current and the voltage) can be actually monitored. In particular it is possible to retrieve the current at which the passage from the superconducting state to the finite voltage occurs in JJs, usually called the switching current (SC). Repeating the measurements in the presence of a random disturbance, or because of quantum effects [3], the junction can switch to the finite voltage at slightly different current levels, thus producing a distribution of SCs. Conversely, the analysis of the distribution of the SCs can be used to reveal the presence of noise or quantum effects. In fact, the analysis of the SCs cumulants have been employed to detect and quantify noise [4-6], or to ascertain its features [7]. In this context, it is attractive the idea to use a $\mathrm{JJ}$ as a device that is capable to 
discern the non-Gaussian, in particular Poissonian, character of the non-intrinsic noise [8].

We propose to extend these ideas to graphene-based JJs affected by Lévy noise, that is non-Gaussian fluctuations characterized by the so-called Lévy flights [9-11], exploiting the information content of the SC distributions, in analogy to the case of driven JJs [12, 13]. There are several reasons to do so. From a general point of view, stable non-Gaussian noise sources are interesting exceptions to the general rule that noise is characterized by finite variance. It is therefore valuable to have a tool that can accurately discriminate the presence of small amounts of 'fat tails' disturbances to get an estimate of their contribution in the noise background. Moreover, JJs as detectors of non-Gaussian sources offer the advantage that, being superconducting devices, they produce little thermal noise, for the intrinsic resistance of the junction is low and the temperature can be decreased as much as it is necessary or possible. JJs are therefore well suited to be used as on chip detectors of current fluctuations to characterize a noise source that feeds the junction. The interest also resides in material issues, for it has been noticed [14] that graphenebased JJs can exhibit anomalous SCs with premature switches. Early escapes suggest the presence of anomalous disturbances, that are likely to be unrelated to thermal fluctuations. Recently, it has also been proposed that the particular electron-electron interaction of the graphene electronics can produce a peculiar response to a laser source, namely, a Lévy flights distribution [15]. Yet, a graphene stripe with anisotropically distributed on-site impurities is demonstrated to reveal Lévy flight transport in the stripe direction [16]. Therefore, a graphene based device in this configuration could be at the same time both the source of the noise and the detector to reveal its presence. This work has been prompted by the observation that Lévy noise has been postulated only in the specific case of graphene-based JJ [1416], and that to reveal its presence might be of particular relevance for material issues that pertain graphene.

Determining the properties of the Lévy noise from the promptly available data, the SC distributions, offers a qualitative advantage: the data can be indeed collected in a preestablished time, because the bias ramp gives a maximum time for each point, $T=1 / v_{\mathrm{b}}$. By contrast, according to the nature of the Lévy flights, the extreme fluctuations that cause the flat tails of the Lévy noise distributions are rare but essential to characterize the tails; therefore, exceedingly long expectation times are usually required to build up a robust statistical analysis. The special bias scheme to record SC, that guarantees that the data are collected in a maximum finite time $1 / v_{\mathrm{b}}$, that is determined by the experimental set-up, is shown in the inset of figure 1(a).

To implement JJs as noise detectors, however, requires a careful analysis [17-23]. Lévy flight sources are expected to induce a distortion of the cumulants of the SCs distribution (with respect to the Gaussian case) [17]. Here we propose to go beyond the analysis of the cumulants, to retrieve the properties of the noise exploiting the full information content of the SCs distribution. The direct analysis is more convenient, especially to quantify the amplitude of the Lévy noise. (a)

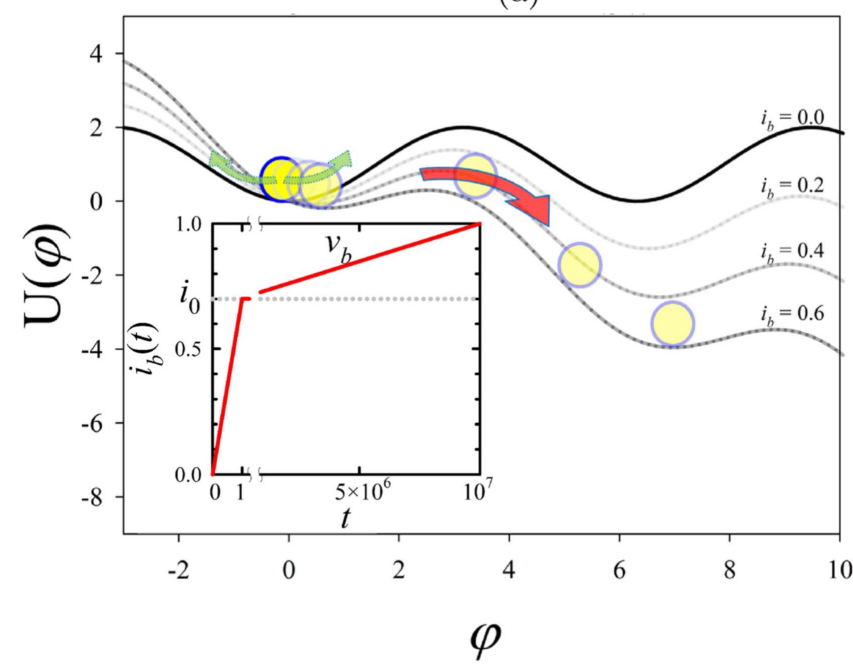

(b)

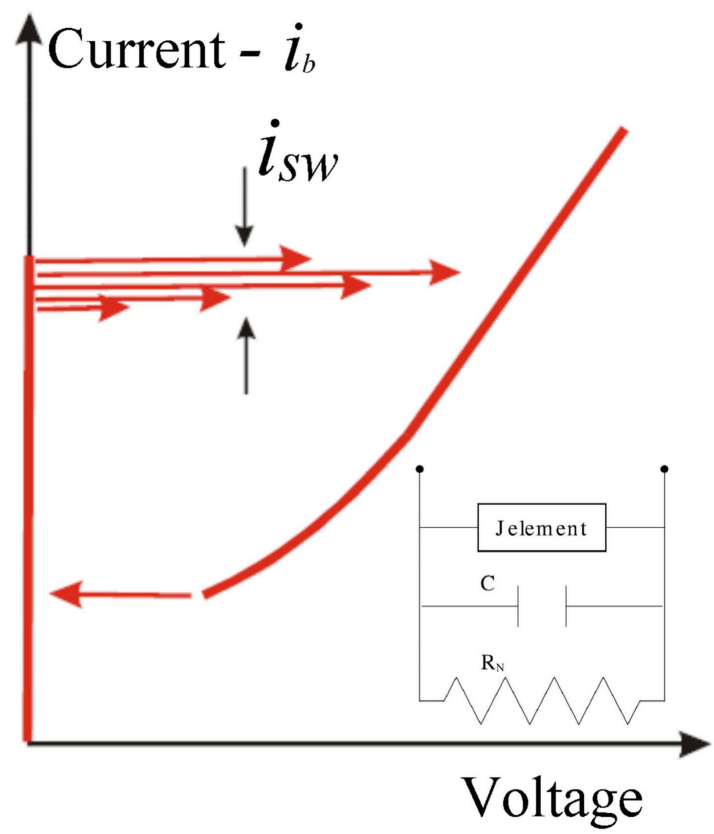

Figure 1. (a) Mechanical analog of a JJ, corresponding to the representative point of the system running down along the washboard potential as the bias current is increased. Due to noise induced fluctuations, the overcoming of the potential barrier occurs in correspondence of a bias current $i_{\mathrm{b}}<1$. In inset: schematic of the bias current sweeping method. The current is fast swept until the initial bias $i_{0}$, and then slowly swept (at a rate $v_{\mathrm{b}}$ ) up to the critical value $i_{\mathrm{b}}=1$. The time scale of the inset is normalized to the inverse of the plasma angular velocity $\omega_{\mathrm{p}_{0}}$, see equation (6). Notice the broken axis, that is necessary to visualize the initial fast ramp. (b) $I-V$ scheme: the bias (vertical axis) is ramped, and the current at which a passage to the finite voltage occurs is recorded. The switching current corresponds to the passage over the maximum of the potential shown in the panel (a). In inset: the electrical equivalent of a JJ.

The work is organized as follows. In section 2 we set the stage for the analysis, discussing the basic equations that govern the system. In section 3 we discuss the methods to analyze the SC resulting from the noisy system and we collect the results of simulations. Section 4 concludes. 


\section{The model}

We investigate a dissipative, current biased short JJ, within the resistively and capacitively shunted junction model. For the normalized current, the basic equation reads [24]

$$
\frac{\mathrm{d}^{2} \varphi(t)}{\mathrm{d} t^{2}}+\beta_{\mathrm{J}} \frac{\mathrm{d} \varphi(t)}{\mathrm{d} t}+i_{\varphi}(t)=i_{\mathrm{f}}(t)+i_{\mathrm{b}}(t)
$$

Here, the currents $i$ are normalized to the critical current of the contact $I_{\mathrm{c}}, \beta_{\mathrm{J}}$ is the standardized friction parameter, time is normalized to the inverse of the plasma frequency $\omega_{\mathrm{p}_{0}}^{-1}=\left(\hbar C / 2 e I_{\mathrm{c}}\right)^{1 / 2}$, where $C$ is the contact capacitance, $\hbar$ is the reduced Planck constant, and $e$ is the electron charge.

This governing equation consists of three parts: (i) the Josephson elements at the left hand side, namely, the capacitive term, the dissipative contribution, and the Josephson supercurrent $i_{\varphi}$, (ii) a noise source $i_{\mathrm{f}}(t)$, and (iii) the external current bias $i_{\mathrm{b}}(t)$. In the following we describe these components.

\subsection{The graphene based Josephson elements}

In a traditional tunnel junction the Josephson normalized current contribution of equation (1), $i_{\varphi}$, is governed by the nonlinear Josephson element

$$
i_{\varphi}(t)=\frac{I(\varphi)}{I_{\mathrm{c}}}=\sin [\varphi(t)] .
$$

A short ballistic graphene-based JJ exhibits a more complicated current-phase relation, that, in the low temperature regime, i.e., $T \lesssim T_{\mathrm{c}} / 4, T_{\mathrm{c}}$ being the critical temperature of the junction, reads $[21,25,26]$

$$
i_{\varphi}(t)=\frac{I(\varphi)}{I_{\mathrm{c}}}=\frac{2}{1.33} \cos \left(\frac{\varphi}{2}\right) \tanh ^{-1}\left[\sin \left(\frac{\varphi}{2}\right)\right] .
$$

The critical current $I_{\mathrm{c}}$ is connected with the junction parameters, namely, the superconductive excitation gap $\Delta_{0}$ and the length and width of the junction $L$ and $W$, respectively, through [25]

$$
I_{\mathrm{c}}=1.33 \frac{e \Delta_{0}}{\hbar} \frac{W}{\pi L}
$$

It is convenient to introduce the mechanical analogous, in which a graphene-based $\mathrm{J}$ is assimilated to a representative point on a potential derived from the peculiar current-phase relation $(3)[21,27]$

$$
\begin{aligned}
U(\varphi, t)= & -E_{\mathrm{J}_{0}}\left\{-\frac{2}{1.33}\right. \\
& \times\left\{2 \sin \left(\frac{\varphi(t)}{2}\right) \tanh ^{-1}\left[\sin \left(\frac{\varphi(t)}{2}\right)\right]\right. \\
& \left.\left.+\ln \left[1-\sin ^{2}\left(\frac{\varphi(t)}{2}\right)\right]\right\}+i_{\mathrm{b}}(t) \varphi(t)\right\},
\end{aligned}
$$

$E_{\mathrm{J}_{0}}=\hbar I_{\mathrm{c}} / 2 e$ being the Josephson energy. It is the potential (5) that characterizes the graphene-based JJ and that specifically distinguishes the JJ with respect to the washboard potential of traditional JJ [24].
The small oscillation frequency reads [21, 27]

$$
\omega_{\mathrm{p}}=\frac{\omega_{\mathrm{p}_{0}}}{\sqrt{1.33}} \sqrt{1-\sin \left(\frac{\varphi_{\min }}{2}\right) \tanh ^{-1}\left[\sin \left(\frac{\varphi_{\min }}{2}\right)\right]},
$$

where $\varphi_{\min }$ is the phase value at a minimum of $U(\varphi, t)$. In the mechanical interpretation, $\omega_{\mathrm{p}}$ is the natural frequency of the system that in the Josephson jargon is named plasma frequency.

In the formulation of the potential (5) it is assumed that the bias current $i_{\mathrm{b}}(t)$ depends upon the time, but its dependence is slow with respect to the JJ timescale $\omega_{\mathrm{p}_{0}}^{-1}$. Next section 2.2 is devoted to the effect of noise in this adiabatic approximation, while the effect of the bias current will be discussed in section 2.3.

The circuit model behind equation (1), see the inset of figure 1(b), consists of a Josephson tunnel current element in parallel with a capacitor and a resistance. The coefficients of the linear elements of equation (1) are normalized so that the capacitance reads 1 , and the resistive term is governed by the damping parameter $\beta_{\mathrm{J}}$

$$
\beta_{\mathrm{J}}=\frac{1}{\omega_{\mathrm{p}_{0}} R_{\mathrm{N}} C}=\beta_{\mathrm{c}}^{-1 / 2},
$$

where $R_{\mathrm{N}}$ is the normal resistance and $\beta_{\mathrm{c}}$ is the McCumber parameter [24]. The dissipation in the nonlinear system slightly alters the proper resonance in equation (6) [24]

$$
\omega_{\text {res }}=\omega_{\mathrm{p}}\left\{\sqrt{1+\beta_{\mathrm{J}}^{2}}-\beta_{\mathrm{J}}\right\} .
$$

\subsection{The noise source}

The term $i_{\mathrm{f}}(t)=I_{\mathrm{f}}(t) / I_{\mathrm{c}}$ in equation (1) represents a random contribution due to the current noise, normalized to the critical current $I_{\mathrm{c}}$. For the Lévy noise the stochastic model is obtained with the approximated finite independent increments [28]. The random current is modeled as a mixture of a standard Gaussian white noise and a Lévy process. This contribution has been proposed to model anomalies in the transport properties, as those described in [15]. Thus, even if the methods described in this work could be in principle applicable to generic JJs, the special form of the noise that we investigate has been, so far, only postulated in graphenebased JJs.

When both Gaussian and Lévy flight fluctuations are considered, the stochastic independent increments read

$$
\Delta i_{\mathrm{f}} \simeq\left(\gamma_{\mathrm{G}} \Delta t\right)^{1 / 2} N(0,1)+\left(\gamma_{\mathrm{L}} \Delta t\right)^{1 / \alpha} S_{\alpha}(1,0,0) .
$$

Here the symbol $N(0,1)$ denotes a normal random variable with zero mean and unit standard deviation. Furthermore, $S_{\alpha}(1,0,0)$ denotes a standard $\alpha$-stable random Lévy variable [23]. These distributions are symmetric around zero, and for $\alpha<2$ are characterized by an asymptotic long-tail power law behavior with exponent $-(1+\alpha)$, while the limit $\alpha=2$ case corresponds to the Gaussian distribution. Moreover, the $\alpha=1$ case corresponds to the well-known Cauchy-Lorentz distribution. The algorithm used to simulate Lévy noise sources is that proposed by Weron [29] for the implementation of the 
Chambers method [30]. The stochastic dynamics of the system is analyzed by integration of equation (1) with a finitedifference method. The time step is fixed at $\Delta t=10^{-2}$, the maximum integration time is $t_{\max }=10^{7}$, and the number of numerical realizations to produce the SC distributions is $N=10^{4}$.

Let us give some physical considerations on the parameter $\gamma_{\mathrm{G}}$ of equation (9). If we consider the pure white noise case $\gamma_{\mathrm{L}}=0$, the statistical properties of the current fluctuations, in physical units are determined by

$$
E\left[I_{\mathrm{f}}(\tilde{t})\right]=0
$$

and

$$
E\left[I_{\mathrm{f}}(\tilde{t}) I_{\mathrm{f}}(\tilde{t}+\tilde{\theta})\right]=2 \frac{k T}{R_{\mathrm{N}}} \delta(\tilde{\theta}),
$$

where $T$ is the temperature of the system, $k$ is the Boltzmann constant, $E[\cdot]$ is the expectation operator, $\tilde{t}$ and $\tilde{\theta}$ denote physical times. In our normalized units, the same equations become

$$
\begin{aligned}
& E\left[i_{\mathrm{f}}(t)\right]=0, \\
& E\left[i_{\mathrm{f}}(t) i_{\mathrm{f}}(t+\theta)\right]=2 \gamma_{\mathrm{G}}(T) \delta(\theta) .
\end{aligned}
$$

Thus, the amplitude of the normalized correlator can be connected to the physical temperature

$$
\begin{aligned}
\gamma_{\mathrm{G}}(T) & =\frac{k T}{R_{\mathrm{N}}} \frac{\omega_{\mathrm{p}_{0}}}{I_{\mathrm{c}}^{2}}=\frac{2 e}{\hbar} \frac{k T}{I_{\mathrm{c}}} \beta_{\mathrm{J}} \\
& =\frac{k T}{E_{\mathrm{J}_{0}}}=\frac{k T}{I_{\mathrm{c}} R_{\mathrm{N}}}\left(\frac{2 e}{\hbar I_{\mathrm{c}} C}\right)^{1 / 2} .
\end{aligned}
$$

For instance, for a short JJ with a critical current $I_{\mathrm{c}}=0.1 \mu \mathrm{A}$ a normal resistance $R_{\mathrm{N}}=100 \Omega$, a capacitance $C=20 \mathrm{pF}$, one finds that the noise amplitude $\gamma_{\mathrm{G}}=10^{-3}$ corresponds to the temperature $T \sim 150 \mathrm{mK}$. As usual for numerical simulations in normalized units, the reported quantities, as the Gaussian white noise amplitude, should be related to physical quantities through the system physical parameters: the critical current, the normal resistance, the capacitance, and the temperature of the JJ. The correspondence between a temperature in the range of hundreds of $\mathrm{mK}$ and the value $\gamma_{\mathrm{G}}=10^{-3}$ only highlights the connection to exemplify the meaning of the dimensionless quantity $\gamma_{\mathrm{G}}$.

The physical interpretation of the Lévy process component, $\gamma_{L}$ in equation (9), can be reconducted to the Lévy-Ito decomposition theorem [31]. Shortly, this theorem states that Lévy process is a mixture of a pure Poissonian-like jump process and a standard Brownian motion. In the absence of the white noise, $\gamma_{\mathrm{G}}=0$, the current fluctuations consist of finite jumps alternated with a standard Brownian erratic drift.

\subsection{Current source and SC measurements}

In equation (1), the term $i_{\mathrm{b}}$ represents an external bias current normalized to the critical current $I_{\mathrm{c}}$. The essential point to retrieve information on the noise induced activation is to linearly ramp the external bias

$$
i_{\mathrm{b}}(t)=i_{0}+\frac{\mathrm{d} i_{\mathrm{b}}}{\mathrm{d} t} t=i_{0}+v_{\mathrm{b}} t
$$

where $i_{0}$ is an initial bias that can be reached with a very fast initial sweep (see the inset in figure 1(b)). Above $i_{0}$, the current is increased at the speed $v_{\mathrm{b}}$. According to equation (5), when the bias increases, the potential $U$ is tilted, and the trapping energy barrier $\Delta U$ decreases (see figure 1(a)) up to vanish for $i_{\mathrm{b}}=1$. During this process, fluctuations induced by the noise source $i_{\mathrm{f}}$ eventually cause a passage over the top of the potential towards the running state, where a finite voltage $V=(\hbar / 2 e)\langle\mathrm{d} \varphi / \mathrm{d} t\rangle$ appears [1, 2] (see figure 1(b)). Schematically, the experimental procedure is as follows. Above the crossover temperature [32] $T_{\text {co }}^{\star}=\hbar \omega_{R} / 2 \pi k$, the quantum tunnel is negligible and the potential (5) is tilted at a rate $\mathrm{d} i_{\mathrm{b}} / \mathrm{d} t=v_{\mathrm{b}}$ until the representative point of the system 'runs down', i.e. it assumes a sizable speed that, in the Josephson counterpart, corresponds to a time phase variation producing a nonzero mean voltage across the junction (see figure 1(a)). At this point the corresponding bias value $i_{\mathrm{b}} \equiv i_{\mathrm{SW}}$ is recorded (see figure $1(\mathrm{~b})$ ). Without noise this SC reads $i_{\mathrm{SW}}=1$ (that is, the very meaning of the critical current $I=I_{\mathrm{c}}$, the current at which the static state is unstable), while in the presence of fluctuations the switch occurs for $i_{\mathrm{SW}, j}<1$, because of the presence of a random term $i_{\mathrm{f}}$. However, repeating the process, i.e. ramping again the bias current from $i_{\mathrm{b}}(0)=i_{0}$ the measured SC is different, for the random nature of $i_{\mathrm{f}}$. This produces a distribution of the SCs, $i_{\mathrm{SW}, j}$, where the index $j=1, N$ denotes the $j$ th experiment (or realization).

The method of current tilting described in this section acts on a special feature of graphene-based JJs, the potential (5), that is specific of graphene-based JJs. Also, the method of SC analysis is particularly suitable, or perhaps strictly necessary, for nanodevices as those based on JJ, inasmuch the voltage corresponding to the critical current is a macroscopic signature of nanoscale features. Thus, the SC analysis, carried out on graphene-based JJs characterized by the potential (5), allows to investigate a nanoscale device through the analysis of a macroscopic, accessible quantity: the SC.

The distribution of the SCs is the quantity that we employ to characterize the noise features. That is, we analyze the SC data resulting from the above described procedure, and for instance shown in figure 2. This analysis is the subject of the next section.

\section{Switching current analysis}

Let us suppose that the data are collected in the form of SC distributions as those of figure 2. Our objective is to determine the most effective methods to retrieve the noise features in graphene-based JJs.

\subsection{Analysis of the escapes for the Lévy noise case}

In figure 2 the SC distributions, computed in the presence of both Gaussian and Lévy noise contributes, are shown for several values of the Lévy noise amplitude and Lévy parameter $\alpha$, while the thermal noise amplitude is set to $\gamma_{\mathrm{G}}=10^{-3}$. We observe that: (i) if $\alpha$ is fixed, as $\gamma_{\mathrm{L}}$ grows the Lévy noise effects are enhanced, so that the low-current tail of 


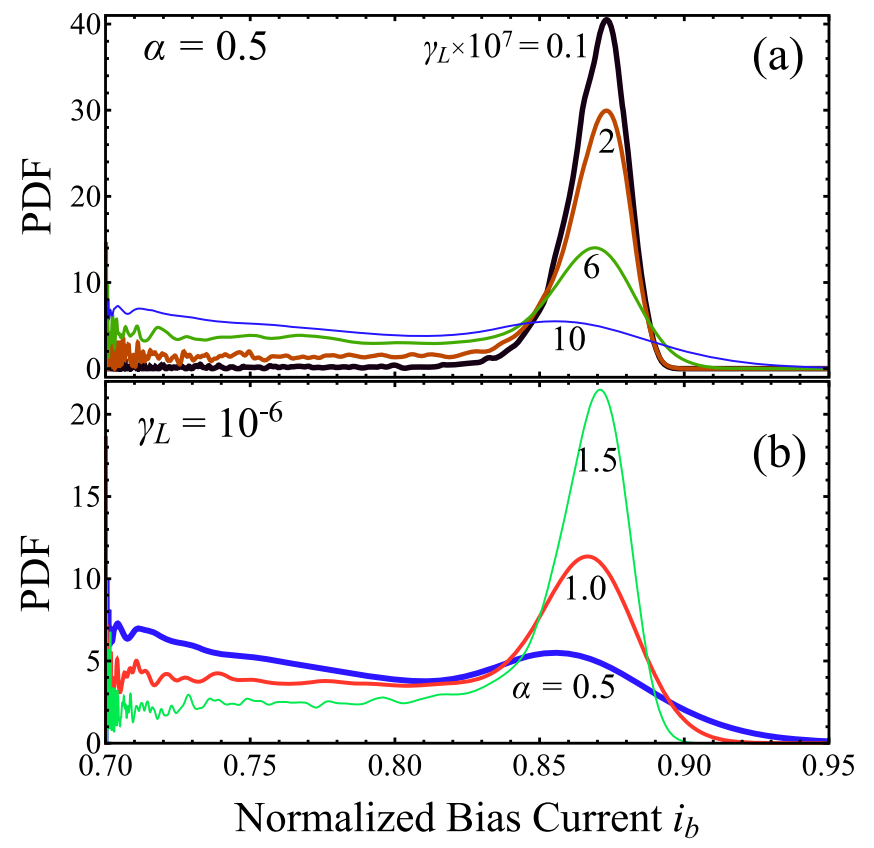

Figure 2. Switching current distributions for several Lévy noise amplitudes (from the highest tail to to lowest) $\gamma_{\mathrm{L}}=10^{-8}, 2 \times 10^{-7}$, $6 \times 10^{-7}, 10^{-6}$ and $\alpha=0.5$, and for different values of the Lévy parameter (from the highest tail to the lowest) $\alpha=0.5,1.0,1.5$ and $\gamma_{L}=10^{-6}$, see panels a and $b$, respectively. The parameters of the system are: thermal noise amplitude $\gamma_{\mathrm{G}}=10^{-3}$, initial bias $i_{0}=0.7$, damping parameter $\beta_{\mathrm{J}}=0.10$, and ramping speed $v_{\mathrm{b}}=10^{-7}$. The current is ramped at a speed $v_{\mathrm{b}}=10^{-7}$.

the SC distributions raises, while the peak decreases, see figure 2(a); (ii) if the $\gamma_{\mathrm{L}}$ value is fixed, as $\alpha$ increases the lowcurrent tail of the $\mathrm{SC}$ distributions is depleted and the peak grows (see figure 2(b)). The position of the peak of the SC distributions is only slightly affected by the change of the Lévy parameters, thus underlining the thermal origin of this spike.

Escapes over a barrier in the presence of Lévy noise have been extensively investigated for the overdamped case $[10,11,33-35]$. It has been found that the asymptotic behavior of the mean escape time under the effect of the Lévy noise is of power-law type $[9,10,35]$. In fact, in the low noise intensity regime, the fat-tail property of the Lévy noise allows for large outliers that dominate the escape process [35]. Put it in other words, the fluctuations corresponding to the extreme values of the Lévy distribution determine the majority of the switches and therefore, in this regime, the escape probability is independent of the energy barrier. The assumption of a switching time $\tau$ independent of the bias current is consistent with the experimental results of Coskun et al [14] that the switching rate for low currents deviates from the expected exponential behavior and shows a plateau (see figure 2(d) of [14]). Accordingly, following [36] the probability density function of the SC, conditioned by the initial bias current $i_{0}$, can be assumed to be of the type

$$
P\left(i_{\mathrm{b}} \mid i_{0}\right) \propto \exp \left[-\mathcal{K} i_{\mathrm{b}}\right]
$$

where $\mathcal{K}$ is a constant that depends upon the noise features.

\subsection{Analysis of the Lévy noise properties through the moments}

If the SC distributions are used to highlight the presence of Lévy noise, a natural solution is to employ the moments of the SC distribution, as the skewness

$$
\tilde{S}[X]=\frac{E\left[(X-\mu)^{3}\right]}{E\left[(X-\mu)^{2}\right]^{3 / 2}},
$$

where $X$ represents the random variable of the measurements, and $\mu$ is the expected value. The quantity (16) can be estimated through the measured SCs $i_{\mathrm{SW}, j}$

$$
S=\frac{\left\langle\left(i_{\mathrm{SW}, j}-\left\langle i_{\mathrm{SW}}\right\rangle\right)^{3}\right\rangle}{\sigma^{3}} .
$$

Here $\left\langle i_{\mathrm{SW}}\right\rangle$ is the estimate of the mean SC and, as usual, $\sigma$ is the estimate of the standard deviation of the switching distribution.

Analogously, the fourth moment, the kurtosis, reads

$$
\tilde{K}[X]=\frac{E\left[(X-\mu)^{4}\right]}{E\left[(X-\mu)^{2}\right]^{2}},
$$

and the corresponding measured moment is

$$
K=\frac{\left\langle\left(i_{\mathrm{SW}, j}-\left\langle i_{\mathrm{SW}}\right\rangle\right)^{4}\right\rangle}{\sigma^{4}} .
$$

If the SC of the JJ can be approximated by a linear system, Gaussian noise should result in a Gaussian distribution of the SC, therefore characterized by $S \simeq \tilde{S}[X]=0$ and $K=3$. Unfortunately, even if the JJs were linear systems, the escape time threshold would introduce an intrinsic nonlinearity that deformates the SC distributions. In fact, the theoretical distributions [38] show that $S=-1$ and $K=5$, thus underlining the nonlinear character of the escape measurements. This has been confirmed in graphene-based junctions and ultrathin superconducting nanowires. In fact Murphy et al [37] have experimentally found for the SC distributions the values $S \approx-1$ and $K \approx 5$.

We go one step further, hypothesizing that deviations from the abovementioned values of the moments $S=-1$ and $K=5$ can be used to quantify the amplitude of the nonGaussian noise input. Numerical simulations of graphenebased $\mathrm{JJ}$ indeed demonstrate that the moments depend upon the noise features, as we show in figure 3. In the figure the intensity of thermal noise is kept fixed to $\gamma_{\mathrm{G}}=10^{-3}$, and the first four moments of the SC distributions show to be dependent on the Lévy noise amplitude. In particular, it is evident that increasing the Lévy noise amplitude $\gamma_{L}$, the mean SC decreases (see figure 3(a)) and the standard deviation increases (see figure 3(b)), for the low current tails of the SC distributions are enhanced (see figure 2). From the results shown in panels (c) and (d) of figure 3 it is evident that the behavior of the higher moments is nonlinear, since the skewness shows a minimum and the kurtosis has a peak for $\gamma_{\mathrm{L}} \sim 2 \times 10^{-8}$. The method can be effectively used to determine the nature of the noise affecting the JJ, but has some limits when employed to measure the non-Gaussian component, i.e. to estimate the coefficient $\gamma_{L}$. In fact, let us 

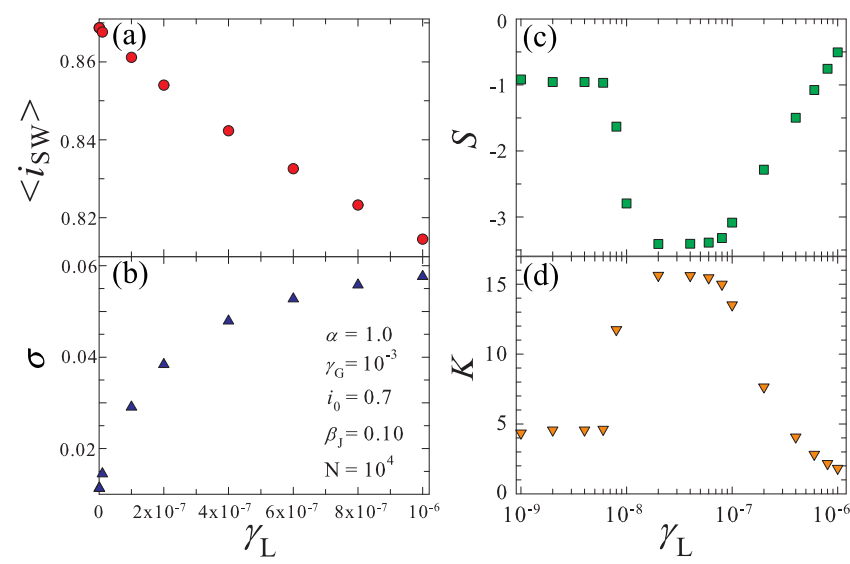

Figure 3. Dependence of the moments upon the Lévy noise intensity $\gamma_{\mathrm{L}}$ for $\alpha=1.0$. We underline that the limiting values for negligible Lévy noise amplitude of skewness and kurtosis agree with the theoretical estimates given in [37]. The parameters of the system are: thermal noise amplitude $\gamma_{\mathrm{G}}=10^{-3}$, initial bias $i_{0}=0.7$, damping parameter $\beta_{\mathrm{J}}=0.10$, and ramping speed $v_{\mathrm{b}}=10^{-7}$.

suppose that one wants to estimate the noise amplitude $\gamma_{\mathrm{L}}$ through experimental measures of the skewness and kurtosis. Because of the non-monotonic behaviors shown in figures 3(c), (d), in close proximity of the minimum (maximum) of the skewness (kurtosis) distribution, a single value of $\gamma_{\mathrm{L}}$ cannot be univocally determined by the measurement of $S(K)$. Furthermore, also in the amplitude regions in which the moments behavior tends to become flat, the estimation of $\gamma_{\mathrm{L}}$ from the knowledge of $S$ and $K$ is not easy, and thus the accuracy of the method becomes poor in this region. This is not surprising, for it is known that the parameter estimate is better performed with statistical tests rather than through the evaluation of the moments. (Such an analysis is the subject of the next section 3.3.)

\subsection{Analysis of the Lévy noise properties through the SC distributions}

If JJs are used to reveal the presence of Lévy noise in graphene based junctions, the starting point is to analyze the response to an unknown random perturbations. We first notice that the Lévy noise alone, as shown in figure 4, produces an exponential dependence in the SC cumulative distribution. This is the counterpart of the PDF behavior at low current in figure 2. In fact the curves obtained by interpolation of the data with an exponential model, the dotted lines of figure 4, are in very good agreement with numerical simulations (solid lines) until the assumption of equation (15) holds, that is in the fat tail region of the PDFs of figure 2. For low bias currents, the exponential behavior is due to the heavy tail character of Lévy noise. For high values of the noise intensity $\gamma_{\mathrm{L}}$, the tails of the Lévy distribution dominate the switching dynamics, resulting in the early passages of the $\mathrm{JJ}$ to a finite voltage. Instead, when the Lévy noise intensity is very low, i.e. $\gamma_{L}=10^{-7}$ in figure 4 , the switches induced by Lévy flights occur at a lower rate. Thus, only in correspondence of high potential slopes, i.e. $i_{\mathrm{b}} \simeq 0.65$ for $\gamma_{\mathrm{L}}=10^{-7}$ in figure 4 ,

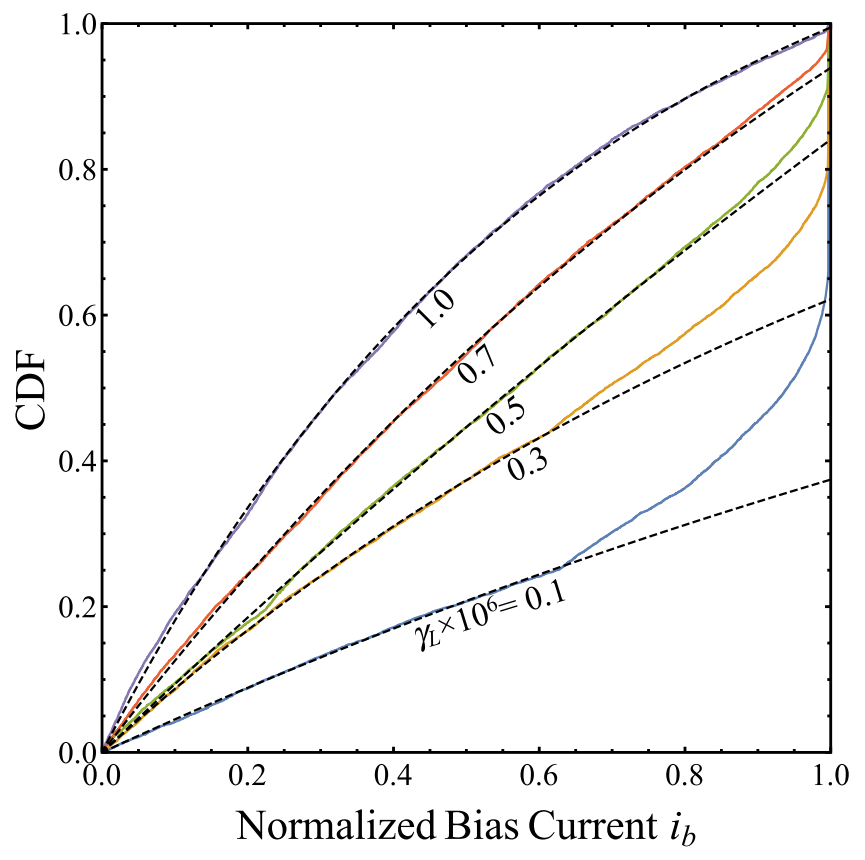

Figure 4. Cumulative SC distributions when only a Lévy noise source, with $\alpha=1$ and $0.1 \leqslant \gamma_{\mathrm{L}} \times 10^{6} \leqslant 1.0$ is considered, i.e. $\gamma_{\mathrm{G}}=0$. The parameters of the system are: initial bias $i_{0}=0.0$, damping parameter $\beta_{\mathrm{J}}=0.10$, and ramping speed $v_{\mathrm{b}}=10^{-7}$. Solid lines represent numerical simulations of equation (1), while dotted lines are the interpolation of the exponential behavior of equation (15).

the peaked behavior of the Lévy noise distribution in the neighborhood of zero induces a steep rise of the cumulative distribution function (CDF).

The detection problem of the Lévy noise presence amounts to retrieve the abovementioned exponential behavior and the values of the parameters $\alpha$ or $\gamma_{\mathrm{L}}$. Having established that Lévy noise gives rise to an exponential behavior, it remains to be devised an effective method to determine the noise properties from the SC.

In doing so, one should first assume that the unavoidable random term, statistically associated to thermal noise, is described by Gaussian distributed fluctuations [24]. If one ascertains the plausibility of some further non-Gaussian disturbances, it is necessary to formulate, on physical grounds, a statistical model for the additional random term. The latter model is the basis to retrieve, with respect to the pure Gaussian hypothesis, the statistical plausibility of the presence of Lévy flights. Thus, if the hypothesis of pure Gaussian noise is rejected on a statistical basis and the Lévy flight hypothesis is acceptable on physical grounds, we propose to retrieve the $\alpha$-stable parameter of the Lévy noise from the analysis of the SC distribution.

To describe the above mentioned procedure in concrete terms, let us begin with simulated SC in the presence of different sources of input noise. The results are collected in figure 5 in the form of $\mathrm{CDF}$; the two panels display the percentage of switching from the superconductive to the normal state that have occurred prior to a certain value of the bias current $i_{\mathrm{b}}$ while repeatedly ramping the current, as per 
(a)

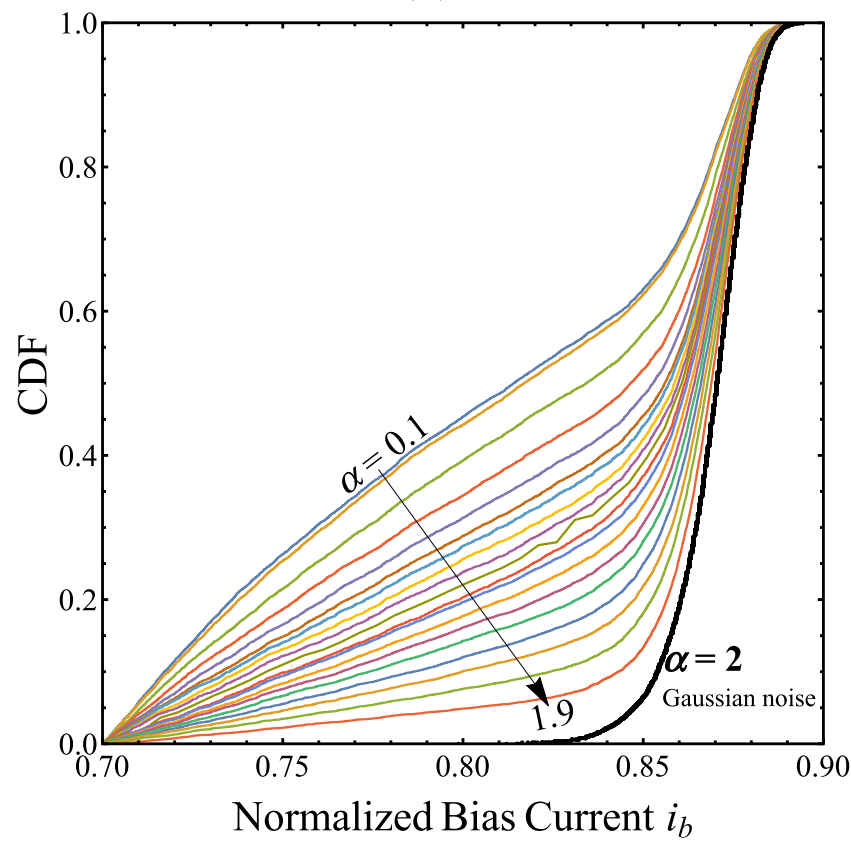

(b)

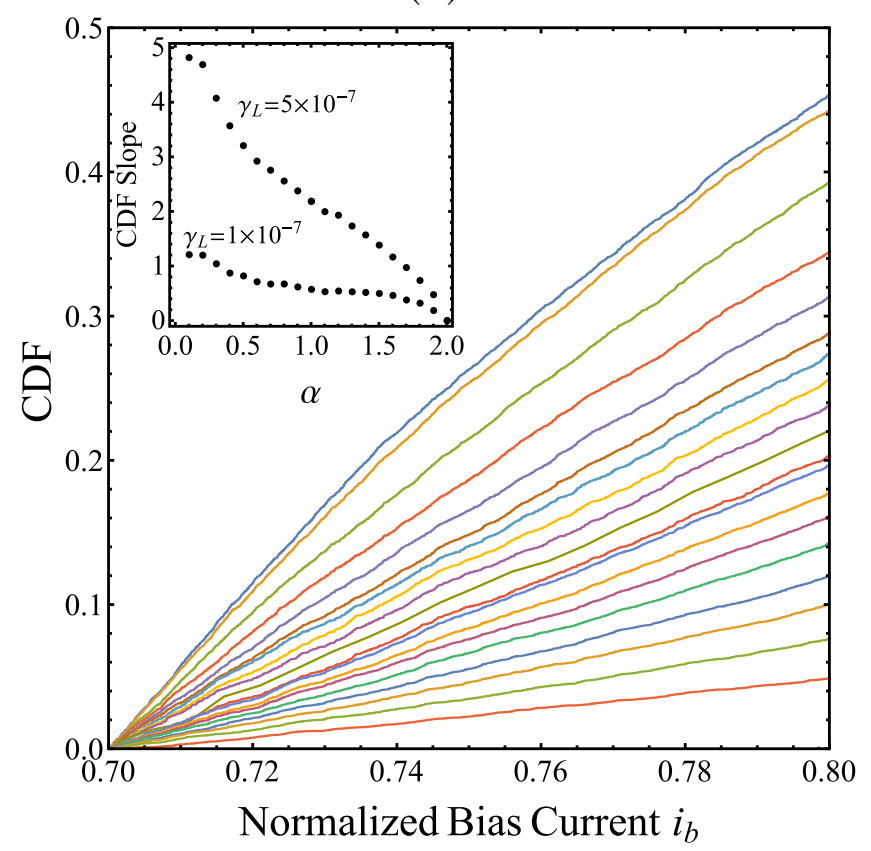

Figure 5. (a) CDFs in the presence of both Gaussian and Lévy noise sources with amplitude $\gamma_{\mathrm{G}}=10^{-3}$ and $\gamma_{\mathrm{L}}=5 \times 10^{-7}$, respectively, and for $\alpha \in(0 \div 2]$ with step 0.1 in the order indicated by the arrow. The Gaussian noise intensity is such that it matches the energy activation for bias $i_{\mathrm{b}} \simeq 0.87$. The other parameters of the system are: initial bias $i_{0}=0.7$, damping parameter $\beta_{\mathrm{J}}=0.10$, and ramping speed $v_{\mathrm{b}}=10^{-7}$. (b) Enlargement of the initial linear part of results in panel (a). In the inset, the slope of the cumulative distribution of the SC as a function of the Lévy distribution parameter $\alpha$ for $\gamma_{\mathrm{L}}=\left(5 \times 10^{-7}, 10^{-7}\right)$.

equation (14). Figure 5 also demonstrates a qualitative finding: the Lévy component gives a clear initial linear slope, that is made evident in the enlargement of figure 5(a) shown in figure 5(b). The change with respect to the pure Gaussian
Table 1. The $p$-values of the KS test as a function of the number of data $N$ for two values of the Lévy noise amplitude $\gamma_{\mathrm{L}}$. The Gaussian noise amplitude is $\gamma_{\mathrm{G}}=10^{-3}$, the Lévy noise parameter is $\alpha=1.9$.

\begin{tabular}{ccc}
\hline$\gamma_{\mathrm{L}}$ & $N$ & $p$ \\
\hline & $10^{2}$ & 0.906 \\
$10^{-7}$ & $5 \times 10^{2}$ & 0.665 \\
& $10^{3}$ & 0.062 \\
& $5 \times 10^{3}$ & 0.004 \\
\hline & $10^{2}$ & 0.813 \\
$5 \times 10^{-7}$ & $5 \times 10^{2}$ & 0.111 \\
& $10^{3}$ & 0.001 \\
& $5 \times 10^{3}$ & $3 \times 10^{-12}$ \\
\hline
\end{tabular}

noise case is a very effective distinctive feature that can be exploited to decide if together with the Gaussian noise there is a Lévy statistics component. In fact, even with very few data, a linear CDF can be effectively distinguished from the zero background for low currents, resulting when only the Gaussian noise contribution is considered.

To detect the $\alpha$ value we show the association between the CDF slope and the Lévy flight parameter $\alpha$ in the inset of figure 5(b). From this figure it is evident that the slope of the cumulative distribution depends upon the parameter $\alpha$ : the smaller $\alpha$ the greater the slope. This is not surprising, because the case $\alpha=2$ corresponds to the Gaussian case, where a neat peak without tails is expected. The change in the slope offers the possibility to determine the parameter $\alpha$.

To make the analysis quantitative, we employ the Kolmogorov-Smirnov (KS) [39] test to distinguish the different outcomes. We have performed the KS test on the data of figure 5, comparing the pure Gaussian case $\left(\gamma_{\mathrm{G}}=10^{-3}\right)$ with the mixture of the same Gaussian source and an additional Lévy noise $(\alpha=1.9)$ of amplitude $\gamma_{\mathrm{L}}=5 \times 10^{-7}$. The result is that the test is very effective, to say the least, as the KS statistics read $D=0.072$. For a sample size of $10^{4}$ $\mathrm{SCs}$, it corresponds to a $p$-value of $10^{-23}$. It is interesting to note that this very high significance is due to a qualitative change between the Lévy flight and the Gaussian noise. In fact, if one compares two Lévy-noise type, say $\alpha=1.3$ with $\alpha=1.4$ (see figure 5), the KS statistics read $D=0.0257$, and the corresponding $p$-value ramps up to $p=0.0026$, about 20 orders of magnitude above. One concludes that the analysis of the SC is particularly effective in detecting the presence of noise of the Lévy type. The KS test also allows to find the minimum number of the measurements to detect the presence of a Lévy noise component (of a given amplitude) with a prescribed $p$-value, or conversely the $p$-value for a given number $N$ of experiments. The results are shown in table 1 , for two values of the Lévy noise amplitude $\gamma_{L}$. The data demonstrate that with a number of experiments in the order of $10^{3}$ it is possible to achieve a $p$-value below $1 \%$. As expected, the lower the noise level, the higher the number of data necessary to confirm the presence of the Lévy noise component. Thus, table 1 can also be used to rule out, for a given 
confidence level $p$, the presence of noise at the amplitude reported.

We conclude that the SCs are potentially interesting to reveal, or to exclude, the presence of an $\alpha$-stable, Lévy noise type, as the switchings are very sensitive to the presence of heavy-tail noise disturbance.

\section{Conclusions}

We have addressed the problem of detecting the presence of non-Gaussian noise in Josephson systems through the analysis of the distribution of the SCs. The question is of relevance in the context of material analysis, specifically for graphene-based JJs where there is the indication that nonequilibrium noise, induced for instance by a laser beam or by anisotropically distributed atoms, can have an infinite, Lévy type, variance. More technically, an infinite variance entails a finite probability that a fluctuation passes any given finite threshold, however large, as the probability of large excursions only decays with an exponent $-\alpha$. We propose a method for analyzing the SC distributions of JJs subject to an unknown source of noise. The method offers some distinct advantages when the noise is characterized by fat tails, i.e. by a finite probability of an infinite fluctuation. This type of noise usually poses a serious difficulty to the experimentalist, for it requires extremely long times to reconstruct the behavior at large values. Thus, to determine the value of the parameter $\alpha$ demands for long experiments (or simulations) to explore extreme values. In contrast, sweeping the bias is very effective, because the bias increase lowers the trapping energy barrier, and therefore in a given ramp time the energy barrier vanishes and a switch event is recorded. The probability of a particle to overcome a barrier when subject to Lévy noise is independent of the barrier height, at least when the ratio between the noise intensity and the energy barrier is high enough [35]. This is remarkably different from the Gaussian noise case, where the probability to overcome the barrier depends exponentially on the barrier energy. Also, if both components contribute to the overall noise level, they do not interfere, because they produce switching at different bias levels: the Lévy noise in the lower part of the distribution, the Gaussian noise when the energy barrier becomes comparable to the noise energy. The practical consequence for the analysis of graphene-based JJs is that the study of the SC distribution is very effective in revealing the presence of Lévy noise. The analysis of the SC moments can be performed to estimate the amplitude of the Lévy noise. It is however more efficient to employ a statistical test, such as the KS test, that can lead to an upper bound for the Lévy noise level. For instance, if the number of the measured SCs is in the order of the thousands, it suffices to rule out at a confidence level of $1 \%$ that the amplitude of the Lévy noise is $10^{-7}$, when the Gaussian noise amplitude is $10^{-3}$, that corresponds, in the normalized units we are using, to about $150 \mathrm{mK}$.

The general conclusion is that the SC distributions in the cases of Lévy and Gaussian noise are remarkably different, and the two cases can be statistically distinguished even if few data are available. We stress that the use of the graphenebased model, especially equation (5), is prompted by the observation that Lévy flight noise has been postulated only in the specific case of graphene-based JJ [14-16], and that to reveal its presence might be of particular relevance for material issues that only pertain graphene.

Apart from material issues for the detection of intrinsic Lévy noise, the efficiency of the method paves the way towards potential applications for the detection of extrinsic noise. We speculate that it could be possible to develop bolometers exploiting graphene-based Josephson detectors, once the system has been adequately calibrated. Such calibration, however, requires a careful analysis of the parameter space, that is, in our view, the priority for the research in this direction.

\section{Acknowledgments}

CG has received funding from the European Union FP7/ 2007-2013 under REA grant agreement no 630925COHEAT and from MIUR-FIRB2013-Project Coca (Grant No. RBFR1379UX). VP acknowledges INFN, Sezione di Napoli (Italy) for partial financial support. DV and BS acknowledge the financial support of the Ministry of Education, University, and Research of the Italian Government.

\section{References}

[1] Josephson B 1962 Phys. Lett. 1 251-253

[2] Josephson B D 1974 Rev. Mod. Phys. 46 251-4

[3] Pierro V and Filatrella G 2016 Phys. Rev. A 94042116

[4] Ankerhold J and Grabert H 2005 Phys. Rev. Lett. 95186601

[5] Peltonen J T, Timofeev A V, Meschke M and Pekola J P 2007 J. Low Temp. Phys. 146 135-59

[6] Timofeev A V, Meschke M, Peltonen J T, Heikkilä T T and Pekola J P 2007 Phys. Rev. Lett. 98207001

[7] Golubev D S, Marthaler M, Utsumi Y and Schön G 2010 Phys. Rev. B 81184516

[8] Pekola J P 2004 Phys. Rev. Lett. 93206601

[9] Dubkov A and Spagnolo B 2007 Acta Phys. Pol. B 38 1745-58

[10] Dubkov A A, Spagnolo B and Uchaikin V V 2008 Int. J. Bifurcation Chaos 18 2649-72

[11] Dubkov A A, La Cognata A and Spagnolo B 2009 J. Stat. Mech.: Theory Exp. P01002

[12] Filatrella G and Pierro V 2010 Phys. Rev. E 82046712

[13] Addesso P, Filatrella G and Pierro V 2012 Phys. Rev. E 85 016708

[14] Coskun U C, Brenner M, Hymel T, Vakaryuk V, Levchenko A and Bezryadin A 2012 Phys. Rev. Lett. 108 097003

[15] Briskot U, Dmitriev I A and Mirlin A D 2014 Phys. Rev. B 89 075414

[16] Gattenlöhner S, Gornyi I V, Ostrovsky P M, Trauzettel B, Mirlin A D and Titov M 2016 Phys. Rev. Lett. 117046603

[17] Grabert H 2008 Phys. Rev. B 77205315

[18] Augello G, Valenti D and Spagnolo B 2010 Eur. Phys. J. B 78 225-34

[19] Guarcello C, Valenti D, Augello G and Spagnolo B 2013 Acta Phys. Pol. B 44 997-1005 
[20] Valenti D, Guarcello C and Spagnolo B 2014 Phys. Rev. B 89 214510

[21] Guarcello C, Valenti D and Spagnolo B 2015 Phys. Rev. B 92 174519

[22] Spagnolo B, Valenti D, Guarcello C, Carollo A, Persano Adorno D, Spezia S, Pizzolato N and Di Paola B 2015 Chaos Solitons Fractals 81 412-24

[23] Guarcello C, Valenti D, Carollo A and Spagnolo B 2016 J. Stat. Mech.: Theory Exp. 054012

[24] Barone A and Paternò G 1982 Physics and Applications of the Josephson Effect (New York: Wiley)

[25] Titov M and Beenakker C W J 2006 Phys. Rev. B 74 041401(R)

[26] Hagymási I, Kormányos A and Cserti J 2010 Phys. Rev. B 82 134516

[27] Lambert J, Carabello S and Ramos R 2011 IEEE Trans. Appl. Supercond. 42716

[28] Chechkin A and Gonchar V 2000 Physica A 277 312-26

[29] Weron R 1996 Stat. Probab. Lett. 28 165-71
[30] Chambers J M, Mallows C L and Stuck B W 1976 J. Am. Stat. Assoc. 71 340-4

[31] Kyprianou A E 2006 Introductory Lectures on Fluctuations of Levy Processes with Applications (Berlin: Springer) ch 2

[32] Grabert H and Weiss U 1984 Phys. Rev. Lett. 53 1787-90

[33] Dybiec B, Gudowska-Nowak E and Hänggi P 2006 Phys. Rev. E 73046104

[34] Dybiec B, Gudowska-Nowak E and Hänggi P 2007 Phys. Rev. E 75021109

[35] Chechkin A V, Sliusarenko O Y, Metzler R and Klafter J 2007 Phys. Rev. E 75041101

[36] Fulton T and Dunkleberger L 1974 Phys. Rev. B 94760

[37] Murphy A, Weinberg P, Aref T, Coskun U C, Vakaryuk V, Levchenko A and Bezryadin A 2013 Phys. Rev. Lett. 110 247001

[38] Garg A 1995 Phys. Rev. B 51 15592-5

[39] Press W, Teukolsky S, Vetterling W and Flannery B 1995 Numerical Recipes vol 1 (Cambridge: Cambridge University Press) 